\title{
Symmetry and configuration in words and patterns of similar structure
}

\section{PAUL N. HAMID, Victoria University of Wellington, Wellington, New Zealand}

Three structural variables, class, symmetry, and configuration, were investigated tachistoscopically. Identification thresholds were significantly lower for ascending configurational stimuli except in the case of symmetrical words. An even stronger relationahip for symmetrical stimuli to have lower thresholds than asymmetrical stimuli was evident. Errors towards symmetry were also found during prethreshold trials being interpreted as the general tendency towards "good form."

Casperson (1950) has suggested that response biases may account for the few studies that have found lower thresholds for "good forms," such as a circle (e.g., Hochberg, Gleitman, \& McBride, 1948). In support of this, Es (e.g., Bridgen, 1933) have reported that at very rapid tachistoscopic speeds, good Gestalten responses are disproportionately frequent. Soltz \& Wertheimer (1959) present a similar explanation for their finding that "good forms" are more easily recognized than "bad" ones. Recent thinking on the New Look in form perception generally agrees with the Gestalt position that variables such as need, set, and frequency, do not affect perception directly. Rather, many threshold parameters evidently act upon the response system instead of the perceptual system. The $\mathbf{O}$ faced with a complex world (or with an ambiguous tachistoscopic stimulus) and relying upon a limited visual system, must supplement his perceptions according to what has happened in the past and whether he can expect it to happen again.

The usual task in response studies has involved recognition of words or nonsense syllables whereas the Gestalt school has typically employed patterned forms composed of dots, line drawings, shapes, and the like. The present study was concerned with both classes of stimuli and a comparison between the two. It involved the identification of simple patterns varying in symmetry and configuration, defined by Havens \& Foote (1964) as the presence of descending, ascending, or flat letters in a word.

Attneave (1953) has shown that the introduction of symmetry may either facilitate or hinder pattern recognition, depending upon the way by which it is introduced. Typically, however, symmetrical patterns were remembered more easily than asymmetrical patterns with the same number of components.

Recent work has demonstrated that structural features of words have a determinative affect on recognition thresholds. Havens \& Foote (1964) have noted, from responses taken immediately preceding complete recognition, that in the firstand especially the middle-letter position of a short English word letter substitutions appeared to be bound by a "form rule." Substitution errors would be made so that certain dimensions of letter structure remained constant, e.g., substituting d's for b's but not g's or a's. Thus the alphabet could be divided into ascending (e.g., b,h,l, etc.), descending (e.g., g,j,p, etc.), and flat (e.g., a,c,e,m, etc.) letters. This suggests that early identification of words lies in the effects of formal features on the repertoire of potential responses. Foote \& Havens (1967) found that five-letter words with descending first and last letters resulted in lower thresholds than similar words with ascending first and last letters, and thresholds for both of these were lower than for words with all letters flat.
How general is this relationship? All the words used by Foote \& Havens (1967) conformed to a symmetry principle, for example, they used the word "front" which could be represented simply as $|ı 1|$. Is the configurational effect a general one or is a symmetry rule involved? If symmetry, at below threshold levels, predominates as a cue for identification then we would expect the pattern II to be more easily identified than 111 as well as "pay" to be more easily identified than "yes." Also, if the symmetry principle predominates at below threshold levels, the descending, ascending, and flat relationships may not hold.

It was therefore hypothesized that: (a) symmetrical patterns and words would result in lower identification thresholds than asymmetrical patterns or words; (b) patterns and words with descending structure would have lower identification thresholds than patterns and words with ascending structure [and this is in line with Foote \& Havens (1967) findings]; and (c) the identification thresholds for words of differing configuration would match those for patterned stimuli of similar structure.

METHOD
Twelve three-letter English words were selected from the Thorndike-Lorge compilation with the following restrictions: they have the same frequency of usage, and differ in configuration and symmetry. A set of figures matching the words for configuration and symmetry were generated by drawing two line sizes, one half the length of the other, in sets of adjacent triplets. A breakdown of the stimuli is shown in Table 1 giving three levels of symmetry, two of configuration, and two of stimulus class.

All words and patterns were placed on $5 \times 9$ stimulus cards ensuring that line size and position in the patterns matched those for words. These were presented tachistoscopically to 15 Ss, in accordance with a previously arranged random sequence for each trial. The stimuli were initially exposed at $40 \mathrm{msec}$ and after each presentation of the stimulus series the exposure time was increased by $10 \mathrm{msec}$ and the stimuli again presented. This procedure was continued until two successive correct reports of the stimulus series were obtained. The set of 12 words was presented first through the entire method of ascending limits, the Ss being required to identify the words or partial words seen. For the form stimuli the same procedure was carried out except that identification was made by the $S$ placing a series of rods into slots in a board to represent the line triplets.

\section{RESULTS}

Identification time data were subjected to an analysis of variance. For both patterns and words symmetry was significant $(F=73.64, \mathrm{df}=2 / 56, p<.001)$. This tendency was more marked for patterns and a significant Class by Symmetry

Table 1

Stimuli According to the Main Variables

\begin{tabular}{llllll}
\hline Configuration & \multicolumn{2}{c}{ Descending } & \multicolumn{2}{c}{ Ascending } \\
\hline Class & Word & Pattern & Word & Pattern \\
\hline Symmetrical & pay & 111 & bed & 1,1 \\
& eye & 11 & who & $1 / 1$ \\
Asymmetrical 1 & egg & 111 & off & 111 \\
& yes & 111 & box & 111 \\
Asymmetrical 2 & gym & 111 & the & 111 \\
& cup & 11 & oil & 111 \\
\hline
\end{tabular}




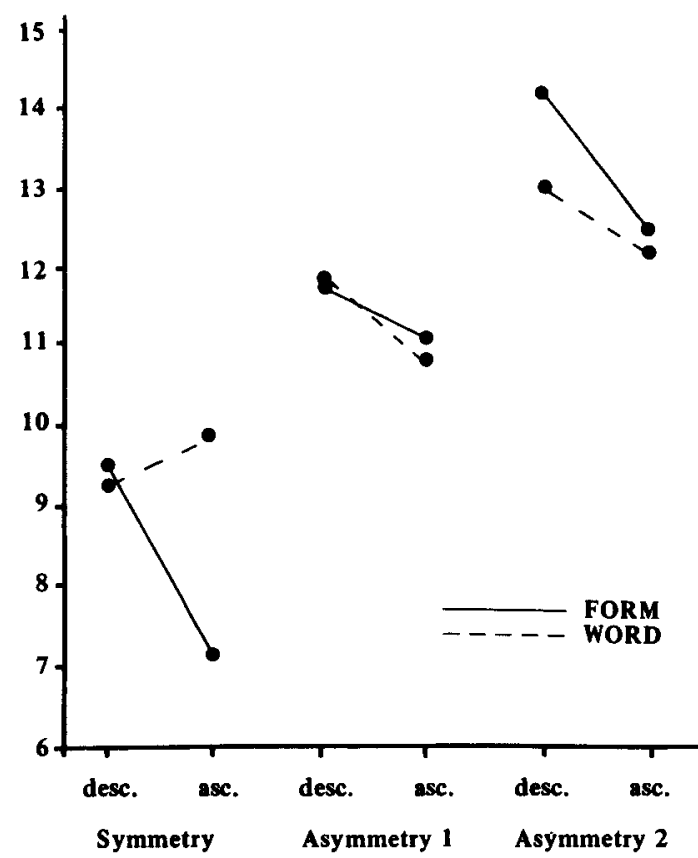

interaction was observed ( $F=5.15, \mathrm{df}=2 / 56, \mathrm{p}<.01)$. Configuration was also significant $(\mathrm{F}=9.97, \mathrm{df}=1 / 28$, $p<.01$ ). A hierarchy was noted; except for the symmetrical word conditions all threshold values were significantly lower for ascending than descending configurations and this was more marked for pattern, hence the significant Class by Symmetry by Configuration $(F=19.73$, df $=2 / 56, p<.01)$ shown in Fig. 1.

A comparison of the percentage of errors made towards symmetry for each $S$ for both patterns and words was tested by means of the Wilcoxon matched-pairs signed-ranks test. The results showed convincing evidence for a general tendency towards symmetry at below threshold speeds (for patterns, $\mathrm{T}=17.5, \mathrm{~N}=15, \mathrm{p}<.02$, and for words, $\mathrm{T}=23.0, \mathrm{~N}=15$, $\mathrm{p}<.02)$.

\section{DISCUSSION}

Symmetrical patterns and words having lower identification thresholds than their asymmetrical counterparts supports previous research and together with the significance of the symmetry effect and the analysis of errors verifies the Gestalt notion of the tendency towards "good form." Important, however, is the fact that not only was this trend evident for relatively meaningless patterns, but it also occurred quite markedly in word stimuli. One might expect large differences in threshold values for words vs patterns due to past experience but there can be no doubt that the results of the present study show that the effect of frequency of prior exposure are minor compared to that of symmetry, under near subliminal conditions.

The prediction that stimuli with descending configuration would have lower thresholds was upheld only in the case of symmetrical word stimuli. All other stimuli produced the inverse of this trend (Fig. 1). It is interesting to note also that this exception was the only combination Foote \& Havens (1967) have tested so that it can be said that their results may only be applicable to this class. Why this exception, is a matter for future research and seems impossible to explain under the present research paradigm. It may be that with words having a symmetrical structure competing tendencies of symmetry markedly reduce configurational effects. The analysis of errors during acquisition for this class of stimuli did show relatively fewer errors towards symmetry.

The degree of match between thresholds for patterns and words is pronounced, indicating the generality of Gestalt principles for word stimuli. Probably the structure of each triplet is more important in determining threshold values than simple similarities or differences between individual components in the set. This agrees with Imai \& Garner (1968) who found, in a series of experiments, attribute structure of total sets or subsets of stimuli more important in determining perceptual classification than simple similarity or difference relations between stimuli. There is, therefore, strong support for the notion of stimulus sets being perceived more in relation to the total set than in contrast to other subsets of the same size. This seems to be true, in the present study at least, for the symmetry dimension.

\section{REFERENCES}

ATTNEAVE, F. Symmetry, information, and memory for patterns. American Journal of Psychology, 1955, 68, 209-222.

BRIDGEN, R. L. A tachistoscopic study of the differentiation of perception. Psychological Monographs: General \& Applied, 1933, 44 (Whole No. 197).

CASPERSON, R. C. The visual discrimination of geometric forms. Journal of Experimental Psychology, 1950, 40, 668-681.

FOOTE, W. E., \& HAVENS, L. L. Differential effects of stimulus frequency and graphic configuration in free- and forced-choice experiments. Journal of Experimental Psy chology, 1967, 73, 340-346.

HAVENS, L. L., \& FOOTE, W. E. Structural features of competitive responses. Perceptual \& Motor Skills, 1964, 19, 75-80.

HOCHBERG, J. E., GLEITMAN, H., \& McBRIDE, P. D. Visual thre shold as a function of simplicity of form. American Psychologist, 1948, 3, 341-342. (Abstract)

IMAI, S., \& GARNER, W. R. Structure in perceptual classification. Psychonomic Monograph Supplements, 1968, No. 9 (Whole No. 25). SOLTZ, D. F., \& WERTHEIMER, M. The retention of "good" and "bad" figures. American Joumal of Psychology, 1959, 72, 450-452. 